\title{
An attempt to find the use of atomic scattering factors and centrosymmetric cubic space groups; two choices of random phases in direct methods
}

\author{
P. S. Yuen \\ 237 Des Voeux Road West, 5th Floor, Hong Kong \\ puisumyuen@netvigator.com
}

In [1], we have used one hydrogen atom with random coordinates in a general position of centrosymmetric cubic space groups to calculate the phases. An approximate structure of the crystal is contained in the peaks of the calculated electron densities. As atomic numbers and atomic scattering factors are not included, the electron densities of the peaks of the approximate structure of calculated electron densities do not follow the pattern of the atomic species. Procedures for identifying this approximate structure are presented in [1]. A simple new method for crystal structure determination is presented in [2]. This involves a large number of combinations of the peaks. If we can distinguish the different types of atoms, the procedures in [1] will be more efficient. The number of combinations in [2] will be significantly reduced. This leads to two fundamental and important questions in X-ray crystallography: What is the result of using atomic numbers and centrosymmetric cubic space groups? What is the result of using atomic scattering factors and centrosymmetric cubic space groups? If we can obtain an answer to one of these questions, the peaks in the calculated electron densities may then be classified into species of atoms. This is very useful for identification of the atoms of the approximate structure. As an attempt to answer the second question, in this article, we use the lightest and heaviest atom of the crystal, with random coordinates in general positions of the space group. As in [1], the result of using phases from these atoms is that an approximate structure of the crystal is embedded in the peaks of the calculated electron densities. The electron densities of the peaks of approximate structure do not follow the trend of atomic numbers. More investigation is needed.

Random initial phases have been employed in direct methods. Phases from one atom with random coordinates in a general position, and phases from the lightest and heaviest atom may be used as random phases. They are random in the sense that they are phases from one or two atoms with random coordinates. They have the property of an approximate structure of the crystal contained in the peaks of the calculated electron densities. This may be useful when these are employed as random phases in direct methods.

[1] Yuen, P. S. Result of using atoms and centrosymmetric cubic space groups. (Unpublished).

[2] Yuen, P. S. Determination of structure of $\mathrm{CoS}_{2}$ by the means of a simple new method; a solution to the phase problem for centrosymmetric cubic crystals. (Unpublished).

Keywords: initial phases; random phases; approximate structure; 